\title{
OPEN A novel model of care for simplified testing of HBV in African communities during the COVID-19 pandemic in Spain
}

\author{
Camila A. Picchio ${ }^{1 凶}$, Daniel K. Nomah ${ }^{2}$, Silvia G. Araujo ${ }^{1}$, Ariadna Rando-Segura ${ }^{3}$, \\ Emma Fernández ${ }^{1}$, Maria Butii, ${ }^{4,5}$, Sergio Rodríguez-Tajes ${ }^{4,6}$, Sabela Lens ${ }^{4,6}$, \\ Francisco Rodríguez-Frías ${ }^{3,4}$ \& Jeffrey V. Lazarus ${ }^{1}$
}

Chronic hepatitis B virus (HBV) infection is a major public health threat for migrant populations in Spain and efforts to scale up testing are needed to reach the WHO elimination targets. The Hepatitis B Virus Community Screening and Vaccination in Africans (HBV-COMSAVA) study aims to use pointof-care testing and simplified diagnostic tools to identify, link to care, or vaccinate African migrants in Barcelona during the COVID-19 pandemic. From 21/11/20 to 03/07/2021, 314 study participants were offered HBV screening in a community clinic. Rapid tests for HBsAg screening were used and blood samples were collected with plasma separation cards. Patients received results and were offered: linkage to specialist care; post-test counselling; or HBV vaccination in situ. Sociodemographic and clinical history were collected and descriptive statistics were utilized. 274 patients were included and $210(76.6 \%)$ returned to receive results. The $\mathrm{HBsAg}$ prevalence was $9.9 \%$ and $33.2 \%$ of people had evidence of past resolved infection. Overall, 133 required vaccination, followed by post-test counselling $(n=114)$, and linkage to a specialist $(n=27)$. Despite the COVID-19 pandemic, by employing a community-based model of care utilizing novel simplified diagnostic tools, HBVCOMSAVA demonstrated that it was possible to diagnose, link to care, and vaccinate African migrants in community-based settings.

If left untreated, chronic hepatitis B virus (HBV) infection can lead to serious liver complications, such as cirrhosis or liver cancer ${ }^{1}$. Currently, an estimated two billion people worldwide have evidence of present or past HBV infection $^{2}$, and estimates further report that 257 million people are currently chronic HBV carriers, of which the majority are living in sub-Saharan Africa (SSA) and South-East Asia ${ }^{3}$. Hepatitis B and hepatitis C virus (HCV) are responsible for more than $50 \%$ of all liver cancer cases, which is the leading cancer cause of mortality globally and the second on the African continent ${ }^{4}$. In West Africa, hepatocellular carcinoma (HCC) is the leading cause of cancer deaths in males and estimates report that $25 \%$ of young adults which have been infected during childhood will die prematurely from chronic HBV complications ${ }^{5}$.

In Spain, the number of migrants arriving from African countries such as Ghana has increased substantially in the last years, and the prevalence of HBV among these migrant groups needs to be addressed by the Spanish public health system ${ }^{6}$. A systematic review and meta-analysis in Ghana reported that the hepatitis B surface antigen (HBsAg) prevalence ranged from 0.55 to $14.3 \%{ }^{7}$ and the risk of being infected with HBV in most subSaharan African countries is estimated to be more than $60 \%{ }^{8,9}$. Given the high prevalence of HBV coupled with the elevated risk of infection acquisition and implementation of the HBV vaccine into the Expanded Programme on Immunization (EPI) only in $2002^{10}$, Ghanaian migrants in Spain are at increased risk of chronic HBV infection and late presentation to viral hepatitis care ${ }^{11}$. The prevalence of HBsAg in Catalonia, where Barcelona is, is estimated at $0.52 \%^{12}$, and $\mathrm{HBV}$ is treated and managed at the specialist level, which requires a referral pathway

\footnotetext{
${ }^{1}$ Barcelona Institute for Global Health (ISGlobal), Hospital Clínic, University of Barcelona, Barcelona, Spain. ${ }^{2}$ Department of Health, Center for Epidemiological Studies on Sexually Transmitted Infections and HIVIAIDS in Catalonia (CEEISCAT), Generalitat of Catalonia, Badalona, Spain. ${ }^{3}$ Liver Pathology Unit, Biochemistry and Microbiology Service, Hospital Universitari Vall d'Hebron, Barcelona, Spain. ${ }^{4} \mathrm{CIBER}$ Hepatic and Digestive Diseases (CIBERehd), Instituto Carlos III, Madrid, Spain. ${ }^{5}$ Liver Unit, Hospital Universitari Vall d'Hebron, Barcelona, Spain. ${ }^{6}$ Liver Unit, Hospital Clínic de Barcelona, IDIBAPS, Barcelona, Spain. ${ }^{\square}$ email: Camila.Picchio@isglobal.org
} 


\begin{tabular}{|l|l|}
\hline $\mathrm{HBsAg}+$ & Refer to specialist care^ \\
\hline $\mathrm{HBsAg}-/$ anti-HBc+ & Past resolved infection, inform of status and provide post-test counselling \\
\hline $\mathrm{HBsAg}-/$ anti-HBc- & If no prior vaccination reported, offer vaccination against $\mathrm{HBV}$ \\
\hline
\end{tabular}

Box 1. Decision algorithm for care in the HBV-COMSAVA study, November 2020-July 2021. ^ Those who were HBsAg + were referred to a specialist irrespective of the HBV-DNA viral load detected on the PSC.

from primary care or another speciality. Current pathways to access HBV care in Spain remain complex and oftentimes unmanageable for migrant populations, particularly for those with irregular migratory status. Subsequently, health service use is lower among migrant populations as compared to the citizens of their host country ${ }^{13}$. In order to eliminate viral hepatitis in Europe, improving care for migrant populations is crucial ${ }^{14}$.

This underutilization of health services can result in late presentation to hepatitis care ${ }^{15}$, leading to delayed treatment initiation and ensuing liver damage progression, including irreversible liver cancer ${ }^{16}$. Point-of-care (PoC) testing and simplified diagnostic tools can facilitate screening and linkage to care to increase engagement and retainment in care ${ }^{17}$.

The aim of the Hepatitis B Virus Community Screening and Vaccination in Africans (HBV-COMSAVA) study is to use PoC diagnostic tools in community settings to identify and link to care or vaccinate African migrants in the greater Barcelona area in Catalonia, Spain.

\section{Methodology}

This is a prospective cohort study (November 2020-January 2022) of adults ( $\geq 18$ years) participating in a West African migrant community-based HBV screening, linkage to care, and vaccination program in the greater Barcelona area, Catalonia, Spain.

Preliminary data of the first seven months of the study (between 21 November 2020 and 3 July 2021) are reported. People attending religious services or community events were offered HBV screening in a "pop-up" clinic model of care (MoC) utilizing a combination of rapid detection tests (RDT) and simplified sample collection strategies. Participating centers were west African migrant faith-based and community organizations in the greater Barcelona area. Measures to reduce the spread of COVID-19 were in place during clinic hours, including mandatory facemask use at all times, hand sanitizing, and physical distancing. People currently on HBV treatment were excluded from the study.

Intervention. On a previously agreed date, the study team would arrive and set up the clinic according to the available space and resources on intervention days $(\mathrm{N}=20)$. Eleven sociodemographic items and twelve clinical history questions were collected verbally and reported in each patient data collection sheet. Trained culturally targeted peer navigators provided education on viral hepatitis prior to the screening and the team together answered all questions from participants. Pre-intervention training included an overview of the study and its objectives, an overview of HBV infection including modes of transmission and prevention. This approach was employed as it has proven vital in other viral hepatitis community-based intervention programs for migrants ${ }^{18,19}$.

Individuals who agreed to participate were offered a RDT (DETERMINE ${ }^{\mathrm{m}} \mathrm{HBsAg} 2$, Abbott Laboratories) to screen for the presence of the HBV surface antigen (HBsAg) and also had a blood sample collected utilizing a plasma separation card (cobas plasma separation card (PSC), Roche Diagnostics), which required $140 \mu$ of blood per spot (total of 3 spots) for analyzing HBV viral load (HBV-DNA) and co-infection with hepatitis D virus (HDV) through the presence of HDV antibodies (anti-HDV) if HBsAg+, or HBV core antibodies (anti-HBc) if HBsAg- (Box 1). Participants were given the option of sample collection via capillary blood from a fingerstick or whole blood collected intravenously. PSCs were left to dry and later transported to Vall d'Hebron University Hospital Laboratory (Barcelona) for analysis 1-5 days after being collected. Sensitivity and specificity of the PSC are very high (>95\%) and previously described ${ }^{20}$.

Participants were informed of the day the HBV-COMSAVA team would return to the same participating center to provide the results of the laboratory analyses and were also given a "return-date" reminder slip. If a person was $\mathrm{HBsAg+}$, a referral to a specialist was provided during the first visit. Patients had the option of choosing between two university hospitals (Hospital Clínic of Barcelona and Hospital Vall d'Hebron) and were seen through an expedited referral process which did not require a prior appointment. Confirmation of diagnosis and treatment eligibility were determined by the hepatologists within the hospital setting and pathway. If a person was HBsAg- and did not have evidence of immunity, the first dose of the HBV vaccine (ENGERIX-B, GlaxoSmithKline Biologicals) was offered in situ free of charge, in addition to a referral for the subsequent doses at their corresponding primary care centers. The patient identifying code (CIP) for the public health system was recorded in order to document these vaccinations in the central health data system. Those who were anti-HBc+ or who reported prior HBV immunization were offered post-test counseling, which included an explanation of their serostatus and HBV awareness.

Access to the public health system. Participants that did not already have a CatSalut health card (documentation that provides access to the Catalan public health system for residents of Catalonia) were offered an expedited processing system to facilitate its obtention. Requirements for inclusion in this expedited pathway included: state of vulnerability, inability to register their address, and health care need. This pathway was offered by the Red Cross of Catalonia in collaboration with the Health Department of Catalonia. 
Data collection. Data were collected from an epidemiological survey, the RDT result, and the laboratory reports. Data from each patient were collected and transferred to a non-person identifiable Excel sheet. Data were introduced using double-entry validation by two independent researchers, and doubts were resolved by examining the original survey results and laboratory reports.

Variables. Sex, year of birth, country of birth and origin, year of arrival in Spain, pregnancy (for women), number of children, education level, and employment status were collected. Information regarding HBV vaccination status, ever being tested for HBV, having undergone surgery in Spain or in another country (if it was performed in a country other than Spain it was specified where), having a family member with HBV and whether that the HBV-positive individual lived in the same household and having a mother with a positive HBV diagnosis were collected as "yes/no/not sure".

Other variables of interest included; having travelled to the African continent in the last year or planning to travel in the next 12 months, having been incarcerated, having engaged in risky situations in the past 6 months (multiple sexual partners, drug use, unprotected sexual encounter), having an STI or HIV diagnosis, having received a transplant, having an inflammatory bowel disease, having chronic renal insufficiency or receiving hemodialysis treatment, having any tattoos or scarring, and having any other illnesses and taking any medication.

Data analysis. The prevalence of HBV infection was calculated for the whole sample and later stratified by sex, level of education, and years since arrival in Spain. Patients who were HBsAg+ were compared to those who were not. Mean, standard deviation and Student's t-test were used for quantitative variables and frequency and chi-squared test were used for qualitative variables where the level of significance was set at $<0.05$. Data were analyzed using StataCorp statistical software: Release $16^{21}$.

Ethical considerations. This study received ethical clearance in 2020 from the Ethical Committee of the Hospital Clínic de Barcelona, Barcelona, Spain (n. HCB/2020/1036). This study was performed in accordance with relevant guidelines and regulations. All participants provided informed written consent. Study information sheets and informed consent forms were available in Spanish and English.

\section{Results}

From 21 November 2020 to 3 July 2021, 314 people were offered an HBV test. Participants who have their second return visit programmed for a future date and have not yet received results $(n=34)$ are excluded from analysis. After removing participants who did not meet inclusion criteria $(n=6), 274$ were included. Reasons for exclusion included currently in care and receiving HBV treatment $(\mathrm{n}=3)$ and not having an RDT nor dried plasma spot (DPS) sample collected $(n=3)$.

Women represented $42.3 \%(\mathrm{n}=116)$ of the sample, participants where predominantly of Ghanaian origin $(92.0 \%, n=252)$ and the mean age was 41.2 years (SD 10.3). Participants who reported $(n=86)$, on average, had 2.3 children (SD 1.4). The average number of years residing in Spain was 11.6 years (SD 6.5) with $27.0 \%(\mathrm{n}=74)$ of participants having arrived within the last five years.

Hepatitis B infection. The overall prevalence of HBsAg+ detected utilizing the RDT was of $9.9 \% \%(n=27)$. Of these, eleven participants (40.7\%) had detectable HBV-DNA viral loads, one (3.7\%) did not have a PSC sample collected and was directly referred, and the remaining $(55.6 \%, n=15)$ were undetectable based on the PSC results. Two people (7.4\%) had co-infection with HDV evidenced by the presence of anti-HDV on PSC. Prior resolved infection was evaluated in all those who were HBsAg- $(n=246)$ and was detected in $33.2 \%(n=91)$ through the presence of anti-HBc. Sociodemographic variables and potential risk factors associated with HBV infection and anti-HBc positivity are described in Table 1.

Men were twice as likely to have HBsAg positivity in comparison to women (18; $11.4 \%$ vs. 9; $7.8 \%$ respectively $(p=0.314)$ ), while the positivity rate by age was almost the same for those who were HBsAg positive and negative (41.4 years vs. 41.2 years, respectively $(p=0.953)$ ). Both differences in sex and age were statistically insignificant. Those who were HBsAg+ had been living in Spain, on average, for a shorter period of time as compared to those who were HBsAg- (10.6 years (SD 6.0) vs. 11.8 years (SD 6.6) $(p=0.376)$ and was not statistically significant. Education level was significantly associated with HBsAg positivity $(p<0.001)$ and both groups primarily completed up to secondary school (81.5\% who are HBsAg positive v. $85.4 \%$ of those who are HBsAg negative).

HBV vaccination. Most of the participants $192 / 274 ; 70.1 \%)$ reported not being vaccinated against HBV followed by not being sure $(40 / 274 ; 14.6 \%)$. The remaining participants reported being vaccinated $(37 / 247 ; 13.5 \%)$ or having one or two out of three doses of the vaccine $(5 / 274 ; 1.8 \%)$.

Cascade of care. Two hundred and ten (76.7\%) participants returned to receive their results during the second community visit. The majority of patients (133; 48.5\%) required vaccination, 114 (41.6\%) were anti-HBc positive or previously vaccinated and required post-test counselling, and $27(9.8 \%)$ were referred to a specialist (Table 2). Overall, the cascade of care for those who required referral or vaccination is described in Fig. 1.

Accessing the Catalan health system. The majority of participants (266; 97.0\%) reported having a CatSalut health card, which provides access to the Catalan public health care system. Eight participants $(2.9 \%)$ requested expedited health insurance cards. Participants received their associated CIP via email and six received 


\begin{tabular}{|c|c|c|c|}
\hline & $\begin{array}{l}\text { Overall } \\
\mathrm{N}=274(\%)\end{array}$ & $\begin{array}{l}\text { HBsAg+ } \\
n=27(\%)\end{array}$ & $\begin{array}{l}\text { Anti-HBc+ } \\
n=91(\%)\end{array}$ \\
\hline Mean age (SD) & $41.2(10.3)$ & $41.4(8.9)$ & $42.3(9.6)$ \\
\hline Women & $116(42.3)$ & $9(33.3)$ & $44(48.3)$ \\
\hline \multicolumn{4}{|l|}{ Years of residence in Spain } \\
\hline $1-5$ & $74(27.0)$ & $8(29.6)$ & $25(27.5)$ \\
\hline $6-12$ & $61(20.3)$ & $7(25.9)$ & $21(23.0)$ \\
\hline $13-20$ & $116(42.3)$ & $10(37.0)$ & $38(41.8)$ \\
\hline $21+$ & $19(6.9)$ & $1(3.7)$ & $6(6.6)$ \\
\hline No data available & $4(1.5)$ & $1(3.7)$ & $1(1.1)$ \\
\hline \multicolumn{4}{|l|}{ Country of birth } \\
\hline Ghana & $252(91.9)$ & $25(92.6)$ & $86(94.5)$ \\
\hline Senegal & $15(5.5)$ & $1(3.7)$ & $5(5.5)$ \\
\hline Cameroon & $2(0.7)$ & $0(0.0)$ & $0(0.0)$ \\
\hline Nigeria & $1(0.4)$ & $0(0.0)$ & $0(0.0)$ \\
\hline Niger & $1(0.4)$ & $0(0)$ & $0(0)$ \\
\hline Mali & $1(0.4)$ & $0(0)$ & $0(0)$ \\
\hline Liberia & $1(0.4)$ & $1(3.7)$ & $0(0.0)$ \\
\hline United Kingdom & $1(0.4)$ & $0(0.0)$ & $0(0.0)$ \\
\hline \multicolumn{4}{|l|}{ Level of education } \\
\hline No schooling & $16(5.8)$ & $3(11.1)$ & $6(6.6)$ \\
\hline Primary school & $43(15.7)$ & $5(18.5)$ & $12(13.2)$ \\
\hline Secondary school & $173(63.1)$ & $14(51.8)$ & $67(73.6)$ \\
\hline University (bachelor, diploma) & $23(8.4)$ & $2(7.4)$ & $2(2.2)$ \\
\hline Vocational training & $10(3.7)$ & $1(3.7)$ & $3(3.3)$ \\
\hline University (master or higher) & $9(3.3)$ & $2(7.4)$ & $1(1.1)$ \\
\hline Ever undergone surgery & $79(30.8)$ & $12(44.4)$ & $25(27.5)$ \\
\hline Of which were not in Spain & $31(39.2)$ & $2(16.7)$ & $8(32.0)$ \\
\hline \multicolumn{4}{|l|}{ Country where surgery was reported to have taken place } \\
\hline Ghana & $26(83.9)$ & $2(100)$ & $6(100)$ \\
\hline Senegal & $2(6.4)$ & $0(0.0)$ & $0(0.0)$ \\
\hline Nigeria & $1(3.2)$ & $0(0.0)$ & $0(0.0)$ \\
\hline Russia & $1(3.2)$ & $0(0.0)$ & $0(0.0)$ \\
\hline United Kingdom & $1(3.2)$ & $0(0.0)$ & $0(0.0)$ \\
\hline STI diagnosis & $4(1.5)$ & $1(3.7)$ & $2(2.2)$ \\
\hline Family member HBsAg+ & $23(8.4)$ & $4(14.8)$ & $9(9.9)$ \\
\hline Mother HBsAg+ & $3(13.0)$ & $2(50.0)$ & $0(0.0)$ \\
\hline HBsAg+ family member living in same household & $8(34.8)$ & $0(0.0)$ & $3(33.3)$ \\
\hline Recent travel to Africa ( $\leq 6$ months) or plans to travel in next 12 months & $136(49.6)$ & $15(55.6)$ & $55(60.4)$ \\
\hline Tattoos or scarring & $3(1.1)$ & $0(0)$ & $3(3.3)$ \\
\hline Ever been incarcerated & $4(1.5)$ & $0(0)$ & $1(1.1)$ \\
\hline
\end{tabular}

Table 1. Baseline sociodemographic characteristics and risk factors of all patients screened and those who are $\mathrm{HBsAg}$ and anti-HBc positive.

\begin{tabular}{|c|c|c|c|}
\hline Patient treatment decisions & Total N & Number and \% of patients who returned to receive results & $\begin{array}{l}\text { Number and \% of patients who accepted intervention among } \\
\text { those who returned to receive results }\end{array}$ \\
\hline Post-test counselling & 114 & $88(77.2 \%)$ & $88(100 \%)$ \\
\hline Anti-HBc+ & \multicolumn{3}{|l|}{$91^{*}$} \\
\hline Self-reported past HBV vaccination & \multicolumn{3}{|l|}{$37^{\star}$} \\
\hline Vaccination required & 133 & $98(73.7 \%)$ & $84(85.7 \%)$ \\
\hline Referral to specialist care & 27 & $24(88.9 \%)$ & $13^{* *}(54.2 .0 \%)$ \\
\hline
\end{tabular}

Table 2. Patient treatment decisions and cascade of care proportions. ${ }^{\star} 14$ participants reported being previously vaccinated and also were anti-HBc positive. ${ }^{* *} 16$ accepted the direct referral to one of the two hospitals linked to the project, of which 12 had a documented first visit with a specialist. Eight participants preferred visiting their own physicians (not the hospitals included in the study) due to distance and transportation issues, of which one had a documented first visit. 


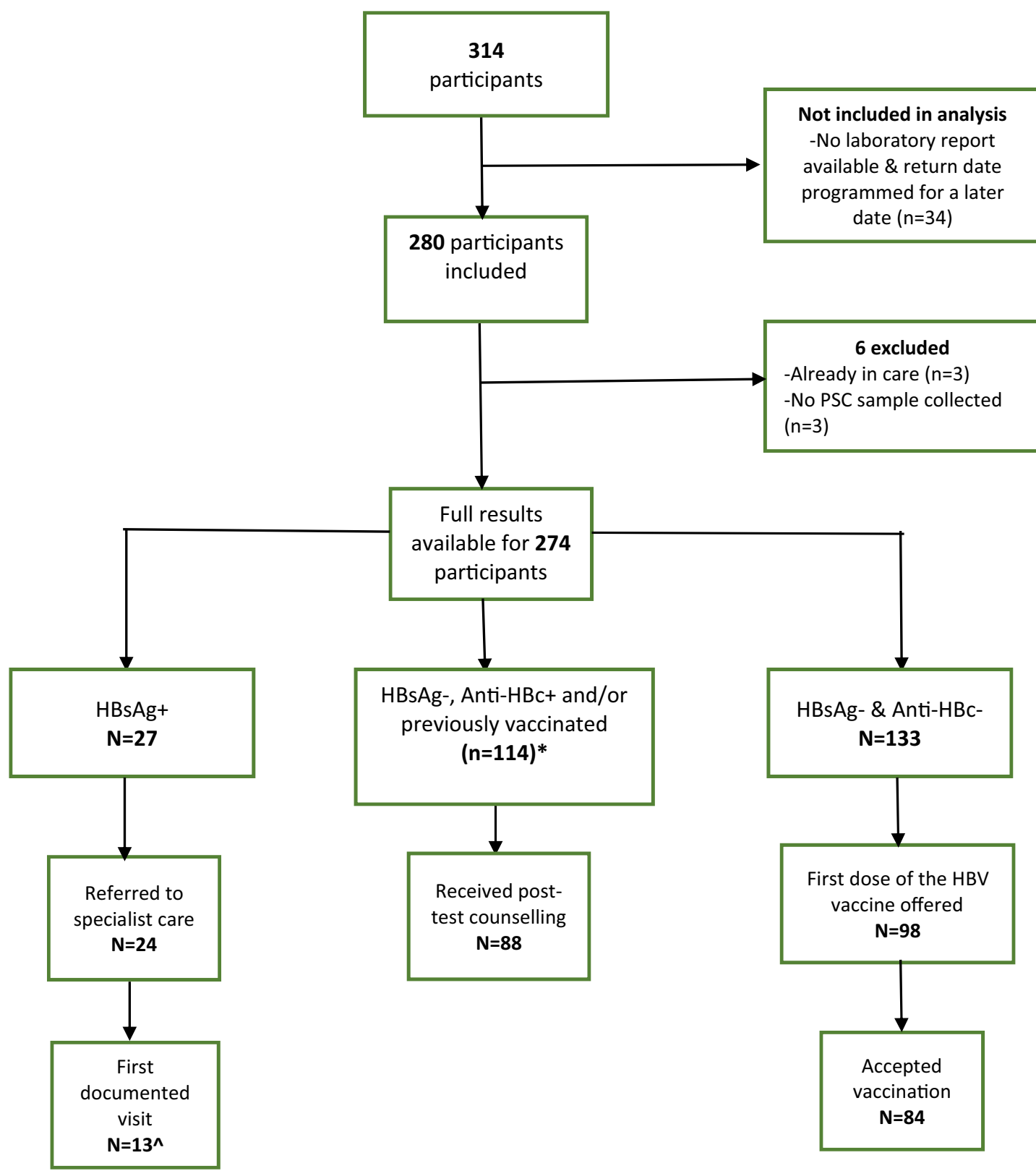

Figure 1. Flow chart of participants in the HBV-COMSAVA study and overall linkage to care proportions. ${ }^{\star}$ Fourteen participants reported being previously vaccinated and were anti-HBc positive. $\wedge 16$ accepted the direct referral to one of the two hospitals linked to the project, of which 12 had a documented first visit with a specialist. Eight participants preferred visiting their own physicians (not the hospitals included in the study) due to distance and transportation issues, of which one had a documented first visit. HBsAg Hepatitis B surface antigen, anti- $H B c$ antibody against hepatitis B virus core antigen, $H B V$ hepatitis $\mathrm{B}$ virus.

the physical card at their home during the project duration. The remaining participants are still waiting for the physical card to arrive but have access to the health system with the CIP number.

\section{Discussion}

The COVID-19 pandemic has disrupted global efforts to curb the transmission of HBV and provide access to care. Difficult to reach and vulnerable populations, such as migrants, are particularly susceptible to these disruptions. Current reports show that about $90 \%$ of HBV services have been hampered by the current pandemic ${ }^{22,23}$. Prior to the COVID-19 pandemic, however, only approximately $5 \%$ of viral hepatitis cases were diagnosed globally ${ }^{3}$, and disruption to diagnostic capacity coupled with limited healthcare access due to over-burdened health systems may further impact HBV screening, treatment initiation and monitoring ${ }^{23,24}$. Missed diagnoses prevent adequate linkage to care and subsequent treatment initiation and monitoring. These missed opportunities can have long-term impact on transmission dynamics. 
Despite the challenges due to the COVID-19 pandemic, by employing a novel dried plasma spot (DPS) test in combination with rapid detection testing, the HBV-COMSAVA model of care demonstrated that it was possible to diagnose, link to care, and vaccinate West African migrants in community-based settings during the pandemic who may otherwise not have received care. Therefore, in order to inform ongoing decision-making about how best to eliminate hepatitis $\mathrm{B}$, preliminary results from this study are being made available.

The high prevalence of HBV infection reported by the HBV-COMSAVA study (9.9\%) highlights the need to target interventions for this at-risk population. These results compare favorably with other studies from Spain ${ }^{25}$, and the United States ${ }^{19}$, which report prevalence rates of 9.6-33.6\% among SSA migrants. In the European Union/ European Economic Area (EU/EEA), the HBsAg prevalence among migrants from endemic countries is estimated at 6\%, and between 1 and 1.9 million migrants born in endemic countries living in the EU are estimated to have chronic HBV infection ${ }^{13}$.

While HBV epidemiology varies by EU country, overall, the prevalence of HBV in the general population is around $1 \%^{26,27}$, and in Catalonia, Spain, the HBsAg estimated prevalence is $0.52 \%{ }^{12}$. However, estimates report that migrant populations residing in Spain could have prevalence rates that are significantly greater in comparison $^{6,13}$.

This significant difference in the rates between West African migrant populations and the Spanish general population reiterates the importance of making HBV prevention, diagnosis, and treatment services more accessible to this key population as (culturally and linguistically) tailored interventions.

More than $95 \%$ of respondents from 34 European countries reported decreased testing volumes for HIV, viral hepatitis, and STI during the COVID-19 pandemic ${ }^{24}$. This impact was reported in community services, non-governmental organizations, and in specialist care. Particularly affected were community services that provide care for key populations. Community-based testing services, both fixed-site and outreach-based, represent potential sites to target specific population groups and individuals who may be at increased risk of infection and who are not in contact with formal health service ${ }^{28}$, as targeted screening campaigns tailored to the local demographics can better reach high-risk populations ${ }^{17}$.

The success of the HBV-COMSAVA model of care is, in part, due to its community-based nature since the team reaches a high prevalence population in settings which are comfortable and known to the people who the program is aimed to assist rather than waiting for patients to reach health care facilities. Evidence in Spain ${ }^{29}$, and other European countries ${ }^{30}$, has reported an underutilization of regular health care services by migrant populations, despite being accessible to them.

Diagnostic tools, like DPS sampling, can simplify the diagnostic process for difficult to reach populations and in resource-limited settings, and dried blood spot testing is recommended for use in these populations by the World Health Organization (WHO) ${ }^{31}$. The use of simplified diagnostic tools with high sensitivity and specificity are a key contribution to scaling up HBV testing. The PSC demonstrated a good concordance with standard serum/plasma samples as an alternative sample type for serological and virological testing used for screening of HBV and HDV infection. However, the limit of detection (LOD) of the techniques employed (serology and nucleic acid amplification tests (NAAT)) are affected by the lesser amount of starting material and dilution of the sample necessary for its reconstitution compared to plasma or serum. Therefore, the markers found in low concentration in plasma are difficult to detect. For this reason, this sample type has an excellent accuracy for the detection of chronically infected patients (HBsAg: sensitivity $97.0 \%$, anti-HBc: sensitivity $97.0 \%$ ) but low accuracy for the detection of resolved infection (anti-HBc: sensitivity $43.8 \%$ ). This same situation occurs when we use NAAT tests. In HBeAg-negative patients, the sensitivity to detect HBV-DNA was low and can be explained by the low HBV-DNA titer of this kind of patients. However, this limitation would not have an impact on selection of patients eligible for HBV therapy; that is, those with HBV-DNA levels > $2000 \mathrm{IU} / \mathrm{Ml}$. In this situation, the sensitivity of PSC increased to almost $96 \%$ and showed a high correlation with conventional venous blood testing ${ }^{20}$.

The HBV-COMSAVA model of care demonstrated how decentralized HBV testing in community-based settings can be another accessible care pathway for hepatitis care for at-risk vulnerable populations. The use of rapid detection testing and novel sample collection methods together with culturally trained peer navigators resulted in increased access to care, including referrals to specialist care or vaccination during the COVID-19 pandemic. This integrated approach can potentially be utilized for other health and social conditions affecting this population, such as non-communicable diseases like diabetes and hypertension ${ }^{32}$, as well as COVID-19 vaccination ${ }^{33}$.

Strategies to increase screening of and vaccination against HBV for migrant populations living in the EU/EEA have shown to be promising and cost-effective ${ }^{34}$, despite highlighted barriers to ensure effective strategies to link patients to care. While our study, overall, had high linkage-to-care proportions, loss to follow-up was reported for some participants requiring linkage to specialist care and vaccination. While positive cases are offered referrals during the first visit to limit patient drop-off, irrespective of their HBV viral load, sociocultural and economic factors such as work hours, transportation costs, and caregiving often times hinder successful linkage to care as evidenced in our $\sim 50 \%$ documented first visit rate. However, our study shows that increased context-specific HBV testing among high-risk migrant populations utilizing novel simplified diagnostic tools is a suitable strategy to increase HBV diagnoses and prevent community-infection through vaccination.

\section{Limitations}

The main limitation of our study includes the LOD of the PSC samples. The LOD of the techniques employed (serology and NAAT) are affected by the lower amount of starting material and dilution of the sample necessary for its reconstitution compared to plasma or serum. For this reason, some patients with low HBV DNA levels $(<1000 \mathrm{IU} / \mathrm{mL})$ were not detected. On the other hand, some patients who were immune to hepatitis B due past resolved infection may not have been detected and were vaccinated unnecessarily. This, however, does not pose a risk to the patient. Additionally, our data may be affected by recall bias as participants may not have remembered 
whether they had received the HBV vaccination specifically or know whether family members are living with hepatitis B. We aimed to mitigate this by asking each participant if they had ever received three shots (a trait specific to this type of vaccine) of the HBV vaccine. Additionally, this is a cross-sectional cohort study so the results are descriptive in nature and the prevalence, for example, may not be generalisable to other West African migrant populations in Spain or elsewhere.

\section{Conclusions}

This real-world demonstration launched during the COVID-19 pandemic showed that a model of care can screen, link to care, and vaccinate migrants for HBV in community settings and ensure access to care through the utilization of RDT and DPS testing. The study reports that close to $50 \%$ of the participants have current HBV infection or a previously resolved infection, which the majority of participants were previously unaware of. Additional studies should be carried out to better understand the barriers to accessing viral hepatitis care for migrants from different high-prevalence countries and ensure adequate linkage to care to prevent loss to followup and to better identify associated risk factors with HBV infection in those communities.

Received: 13 May 2021; Accepted: 6 August 2021

Published online: 25 August 2021

\section{References}

1. World Health Organization. Hepatitis B in the WHO European Region. Fact sheet. July 2017.

2. World Health Organization. Guidelines for the Prevention, Care and Treatment of Persons with Chronic Hepatitis B Infection. WHO Library Cataloguing-in-Publication Data. 2015.

3. World Health Organization. Global Hepatitis Report 2017 (World Health Organization, 2017).

4. Cooke, S. G. et al. Accelerating the elimination of viral hepatitis: A Lancet Gastroenterology \& Hepatology Commission. Lancet Gastroenterol. Hepatol. 4, 135-184 (2019).

5. Lemoine, M., Eholié, S. \& Lacombe, K. Reducing the neglected burden of viral hepatitis in Africa: Strategies for a global approach. J. Hepatol. 62, 469-476 (2015).

6. Calderón, E. S. et al. Hepatitis crónicas virales B y C en población inmigrante en España. Rev. Esp. Salud Publ. 88, 811-818 (2014).

7. Abesig, J., Chen, Y., Wang, H., Sompo, F. M. \& Wu, I. X. Y. Prevalence of viral hepatitis B in Ghana between 2015 and 2019: A systematic review and meta-analysis. PLoS ONE 15(6), e0234348 (2020).

8. Ott, J. J. et al. Global epidemiology of hepatitis B virus infection: New estimates of age-specific HBsAg seroprevalence and endemicity. Vaccine 30, 2212-2219 (2012).

9. Barth, E. R. et al. Hepatitis B/C and HIV in sub-Saharan Africa: An association between highly prevalent infectious diseases. A systematic review and meta-analysis. Int. J. Infect. Dis. 14, e1024-e1031 (2010).

10. Ansa, G. K. et al. Hepatitis B vaccine uptake among healthcare workers in a referral hospital, Accra. Pan Afr. Med. J. 33, 96 (2019).

11. Lazarus, J. V. et al. Too many people with viral hepatitis are diagnosed late-With dire consequences. Nat. Rev. Gastroenterol. Hepatol. 16(8), 451-452 (2019).

12. Rodríguez-Tajes, S. et al. Significant decrease in the prevalence of hepatitis $C$ infection after the introduction of direct acting antivirals. J. Gastroenterol. Hepatol. https://doi.org/10.1111/jgh.14984 (2020).

13. European Centre for Disease Prevention and Control. Public Health Guidance on Screening and Vaccination for Infectious Diseases in Newly Arrived Migrants Within the EU/EEA (ECDC, 2018).

14. Kim, J. U., Ingiliz, P., Shimakawa, Y. \& Lemoine, M. Improving care for migrants is key for viral hepatitis elimination in Europe. Bull. WHO 99, 280-286 (2021).

15. Mauss, S. et al. Late presentation of chronic viral hepatitis for medical care: A consensus definition. BMC Med. 15, 92 (2017).

16. Birke, B. Hepatitis B and C viruses and hepatocellular carcinoma. Viruses 2, 1504-1509 (2010).

17. Lazarus, J. V. et al. Hepatitis C standards of care: A review of good practices since the advent of direct-acting antiviral therapy. Clin. Res. Hepatol. Gastroenterol. 45, 101564 (2021).

18. Perumalswami, P. V. et al. Hepatitis outreach network: A practical strategy for hepatitis screening with linkage to care in foreignborn communities. J. Hepatol. 58, 890-897 (2013).

19. Shankar, H. et al. A novel collaborative community-based hepatitis B screening and linkage to care program for African immigrants. Clin. Infect. Dis. 62(S4), S289-S297 (2016).

20. Martínez-Campreciós, J. et al. Reflex viral load testing in dried blood spots generated by plasma separation card allows the screening and diagnosis of chronic viral hepatitis. J. Virol. Met. 289, 114039 (2021).

21. StataCorp. Stata Statistical Software: Release 16 (StataCorp LLC, 2019).

22. Wingrove, C., Ferrier, L., James, C. \& Wang, S. The impact of COVID-19 on hepatitis elimination. Lancet Gastroenterol. Hepatol. 5(9), 792-794 (2020).

23. Pley, C. M., McNaughton, A. L., Matthews, P. C. \& Lourenço, J. The global impact of the COVID-19 pandemic on the prevention, diagnosis and treatment of hepatitis B virus (HBV) infection. BMJ Glob. Health 6(1), e004275 (2021).

24. Simões, D., Stengaard, A. R., Combs, L. \& Raben, D. The EuroTEST COVID-19 impact assessment consortium of partners. Impact of the COVID-19 pandemic on testing services for HIV, viral hepatitis and sexually transmitted infections in the WHO European Region, March to August 2020. Euro Surveill. 25(47), 2001943 (2020).

25. Salas-Coronas, J. et al. Newly arrived African migrants to Spain: Epidemiology and burden of disease. Am. J. Trop. Med. Hyg. 98(1), 319-325 (2018).

26. Hernando, V., Ruiz-Alguero, M. \& Díaz, A. Análisis de la evolución de la hepatitis B aguda en España, 2008-2018. Boletín Epidemiólogo Semanal 27(4), 43-49 (2019).

27. Hofstraat, S. H. I. et al. Current prevalence of chronic hepatitis B and C virus infection in the general population, blood donors and pregnant women in the EU/EEA: A systematic review. Epidemiol. Infect. 145(14), 2873-2885 (2017).

28. Mason, L. M. K. et al. Hepatitis B and C testing strategies in healthcare and community settings in the EU/EEA: A systematic review. J. Viral Hepatitis 26(12), 1431-1453 (2019).

29. Llop-Gironés, A. et al. Immigrants' access to health care in Spain: A review. Rev. Esp. Salud Publ. 88(6), 715-734 (2014).

30. Sarría-Santamera, A. et al. A systematic review of the use of Health services by immigrants and native populations. Public Health Rev. 37, 28 (2016).

31. World Health Organization. Guidelines on Hepatitis B and C Testing. Policy brief. November 2016. WHO/HIV/2016.23.

32. Agyemang, C. et al. Obesity and type 2 diabetes in sub-Saharan Africans-Is the burden in today's Africa similar to African migrants in Europe? The RODAM study. BMC Med. 14, 166 (2016).

33. Lazarus, J. V. et al. Vaccinate fast but leave no one behind: A call to action for COVID-19 vaccination in Spain. Commun. Med. 1(12), 1-4 (2021). 
34. Myran, D. T. et al. The effectiveness and cost-effectiveness of screening for and vaccination against hepatitis B virus among migrants in the EU/EEA: A systematic review. Int. J. Environ. Res. Public Health 15(9), 1898 (2018).

\title{
Acknowledgements
}

CAP and JVL acknowledge support to ISGlobal from the Spanish Ministry of Science, Innovation and Universities through the "Centro de Excelencia Severo Ochoa 2019-2023" Programme (CEX2018-000806-S), and from the Government of Catalonia through the "CERCA Programme". CAP acknowledges support from the Secretaria d'Universitats i Recerca de la Generalitat de Catalunya and the European Social Fund as an AGAUR-funded $\mathrm{PhD}$ fellow.

\section{Author contributions}

CAP, DKN, and JVL conceived of the model of care. CAP oversaw data collection in the field carried out by CAP DKN, SGA, and EF. Data entry was performed by CAP and SGA. Data analysis was undertaken by CAP and JVL. A processed the PSC samples in the laboratory after being prepared by ARS and CAP under the supervision of FR. MB, SR, and SL received patients through the referral processed and provided support to data entry and analysis by resolving clinical queries. CAP prepared the first draft of the manuscript and received input from JVL, DKN, ARS, SG, EF MB, SR, SL, and FRF. All authors reviewed the full draft of the article, subsequent revisions, and approved the final version for submission.

\section{Funding}

This study was carried out by ISGlobal with competitive funding through the Gilead Sciences global HBV-CARE program (IN-ES-988-5799).

\section{Competing interests}

CAP, DKN, SGA, ARS, EF and FRF have nothing to declare. MB reports advisory fees from Janssen and Gilead Sciences and grants from Gilead Sciences, outside the submitted work. SR reports speaker fees from Gilead Sciences and Abbvie, outside the submitted work. SL reports speaker/advisory fees from Gilead Sciences and Abbvie and grants from Gilead Sciences, outside the submitted work. JVL reports grants, personal fees and other from AbbVie and Gilead Sciences, personal fees from CEPHEID, GSK, Intercept and Janssen, and grants and personal fees from MSD, outside the submitted work.

\section{Additional information}

Correspondence and requests for materials should be addressed to C.A.P.

Reprints and permissions information is available at www.nature.com/reprints.

Publisher's note Springer Nature remains neutral with regard to jurisdictional claims in published maps and institutional affiliations.

\begin{abstract}
(c) (i) Open Access This article is licensed under a Creative Commons Attribution 4.0 International License, which permits use, sharing, adaptation, distribution and reproduction in any medium or format, as long as you give appropriate credit to the original author(s) and the source, provide a link to the Creative Commons licence, and indicate if changes were made. The images or other third party material in this article are included in the article's Creative Commons licence, unless indicated otherwise in a credit line to the material. If material is not included in the article's Creative Commons licence and your intended use is not permitted by statutory regulation or exceeds the permitted use, you will need to obtain permission directly from the copyright holder. To view a copy of this licence, visit http://creativecommons.org/licenses/by/4.0/.
\end{abstract}

(C) The Author(s) 2021 